\title{
Numerical simulation of unsteady flow in stirred tank
}

\author{
Chao Hui ${ }^{1, a}$, Juan $\mathrm{Xu}{ }^{1, b}$ and Tong Liu $^{2}$ \\ ${ }^{1}$ Shandong Provincial Key Laboratory of Ocean Environment Monitoring Technology, Institute of \\ Oceanographic Instrumentation, Shandong Academy of Sciences, Zhejiang Road 28, Qingdao \\ 266001, China \\ ${ }^{2}$ Qingdao University of Science and Technology

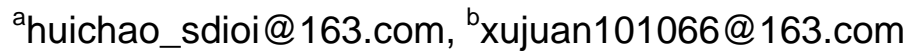

Keywords: ruston turbine; mixing; numerical simulation; unsteady flow

\begin{abstract}
The unsteady flowing property is complicated in stirred tank, so Computational Fluid Dynamics (CFD) is used to simulate the three - dimensional flow in this paper. Taking the steady-state solution as the initial condition of the large eddy simulation, flows in stirred tank are analyzed at different time. The results show that the flow of six-blade ruston turbine is well and velocity distribution is uniform which is good for back-mixing and is unlikely to generate the cavitation. The flow velocity near the blade is obviously higher than that far away from the blade which shows that with the longer distance from the blade, mixing effect is weaker. The low pressure flow characteristic appears in the area near the blade. After squeezed to this area, the high pressure flow is mixed in the blade. The blade whirls to drive the fluid around to form a large number of vortexes. The motion and development of these vortexes enhance the mixing between the materials and the liquid.
\end{abstract}

\section{Introduction}

The stirred tank is one of the common unit equipments in the process industry and it is widely used in chemical industry, biology, pharmacy and the effluent disposal. The flow in the stirred tank is the key factor of efficiency and property in the mixing. Also it is the basic to analyze the mixing, heat transfer and mass transfer. The study found that flow in the stirred tank is very complicated and it is difficult to do the research by the analysis method. The common research methods are experiment and numerical simulation. The normal experimental facility is too expensive to measure the flow of the stirred tank, such as laser doppler velocimeter (LDV) and particle image velocimetry (PIV). While because without such limitation, the numerical simulation method develops rapidly. With the improvement of the computer property and the development of Computational Fluid Dynamics (CFD), there are many superior numerical simulation methods and advanced turbulence models [1-3]. Mature CFD software is used like CFX and FLUENT, which not only shorts the time but also accurately simulates the mixing flow. When numerical simulation is used to research the flow, numerical model reflecting the real flow should be established to simulate the rotate of the stirring paddle, and suitable turbulence model should be chosen. At the same time the boundary condition should be determined. With the large eddy simulation method, unsteady flow of a single six-blade ruston turbine in stirred tank is numerically simulated and analyzed by CFD software FLUENT, which provides guiding basis for further improvement of the mixing equipment.

\section{Governing equation and multiphase flow model}

To improve computational speed, RNG k- $\varepsilon$ model is used, and the preliminary result is taken as the initial condition of the large eddy simulation. Large eddy simulation is the solution of unsteady flow, quadratic implicit formula is used for time discretion and centered difference is used for spatial discretization.

The tubulence energy and dissipation rate equations of RNG k- $\varepsilon$ turbulence model are as follows: 


$$
\begin{aligned}
& \rho \frac{\mathrm{D} k}{\mathrm{D} t}=\frac{\partial}{\partial x_{j}}\left(\alpha_{k} \mu_{e f f} \frac{\partial k}{\partial x_{j}}\right)+2 \mu_{t} \bar{E}_{i j} \frac{\partial \bar{u}_{i}}{\partial x_{j}}-\rho \varepsilon \\
& \rho \frac{\mathrm{D} \varepsilon}{\mathrm{D} t}=\frac{\partial}{\partial x_{j}}\left(\alpha_{\varepsilon} \mu_{e f f} \frac{\partial \varepsilon}{\partial x_{j}}\right)+2 C_{1 \varepsilon} \frac{\varepsilon}{k} v_{t} \bar{E}_{i j} \frac{\partial \bar{u}_{i}}{\partial x_{j}}-C_{2 \varepsilon} \rho \frac{\varepsilon^{2}}{k}-R
\end{aligned}
$$

Where $\mu_{t}$ is eddy viscosity and $v_{t}$ is strain rate tensor. Equivalent viscous coefficient $\mu_{\text {eff }}$ is the sum of coefficient of molecular viscosity $\mu$ and coefficient of eddy viscosity $\mu_{t}$, that is $\mu_{\text {eff }}=\mu+\mu_{t}$.

Large eddy simulation is a medium method between direct numerical simulation (DNS) and Reynolds Averaged Navier-Stokes (RANS). Filter function is used to filter the Navier-Stokes equation in the fourier or spatial domain. LES equation is obtained by filtering the time term. After this filtration treatment, vortexes smaller than filter width or mesh scale can be filtered. Then momentum equation of maelstrom can be obtained. Filter variable is defined as follows.

$$
\phi(X)=\frac{1}{V} \int_{v} \phi\left(X^{\prime}\right) G\left(X, X^{\prime}\right) d X^{\prime}
$$

Where $\Omega$ is the flow field and $G\left(X, X^{\prime}\right)$ is filter function deciding the filter sizes.

The filter operation in the finite volume discretization is as follows.

$$
\phi(X)=\frac{1}{V} \int_{v} \phi\left(X^{\prime}\right) d X^{\prime}, X^{\prime} \in v
$$

Where $\mathrm{V}$ is the volume of computing unit. Filter function $G\left(X, X^{\prime}\right)$ is defined.

$$
G\left(X, X^{\prime}\right)=\left\{\begin{array}{l}
1 / V, X^{\prime} \in v \\
0, X^{\prime} \notin v
\end{array}\right.
$$

Large scale vortex is directly solved and vortex smaller than mesh scale can be solved by subgrid scale model. The traditional subgrid scale model is Smag orinsky-Lilly model, and the model equation is as follows.

$$
\mu_{t}=\rho L_{s}^{2} \bar{S}
$$

Where $L_{S}$ is subgrid mixing length and $S \equiv 2 S_{i j} S_{i j} . L_{S}=\min \left(K d, C_{S} V^{1 / 3}\right)$ in FLUENT and $K$ is Von Karman constant, $d$ is the distance to the recent wall, $C_{S}$ is Smagor insky constant and it is 0.1 in the computation.

\section{Computational model and mesh}

Standard stirred tank is the research subject. The diameter $\mathrm{T}$ is $0.5 \mathrm{~m}$ and the liquid depth $\mathrm{H}$ equals to $\mathrm{T}$. With the six-blade ruston turbine, diameter of the paddle is $\mathrm{D}$, width $\mathrm{W}$ is $\mathrm{D} / 5$, the length $\mathrm{L}$ is $\mathrm{D} / 4$ and the thickness $\mathrm{d}$ is $2 \mathrm{~mm}$. The structure is shown in Fig. 1.

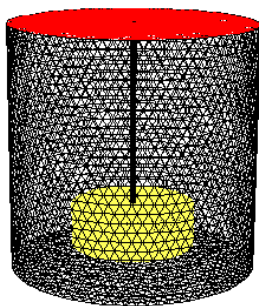

Fig 1 Structure of the stirred tank and schematic diagram of grid

The sliding mesh method combined with hybrid grid method is used in the stirred tank. While in the stirring paddle domain rotating grid and unstructured grid are used. At the same time to better 
description, the meshes are refined. In the other domain, static unstructured grid is used. Because of the sparser mesh, computational burden is reduced to satisfy computational accuracy. The mesh in the thickness direction of the paddle is no less than ten layers, and the number of grid nodes is 489531 . Schematic diagram of grid is shown in Fig.1.

\section{The calculation results and analysis}

After calculating the unsteady flow in the stirred tank, pressure distribution and velocity distribution at different distances from the paddle are analyzed in Fig.2 and Fig.3. Distribution of flow field is good with the average speed $0.56 \mathrm{~m} / \mathrm{s}$ and even distribution of the velocity, which is good for back-mixing and is unlikely to generate the cavitation. It can be seen that when the stirring velocity is low, the cavitation is unlikely to be generated. Then six-blade ruston turbine can be chosen. In fig. 2 along the radial direction, the pressure increases gradually from the inside radically to the outside. In the diameter of the paddle, the pressure increases gradually with the altitude. And pressure near the wall changes small. This demonstrates that the low pressure flow characteristic appears in the area near the blade. After squeezed to this area, the high pressure flow is mixed in the blade. Form the velocity distribution in Fig.3, the flow velocity near the blade is obviously higher than that far away from the blade which shows that with the longer distance from the blade, mixing effect is weaker.

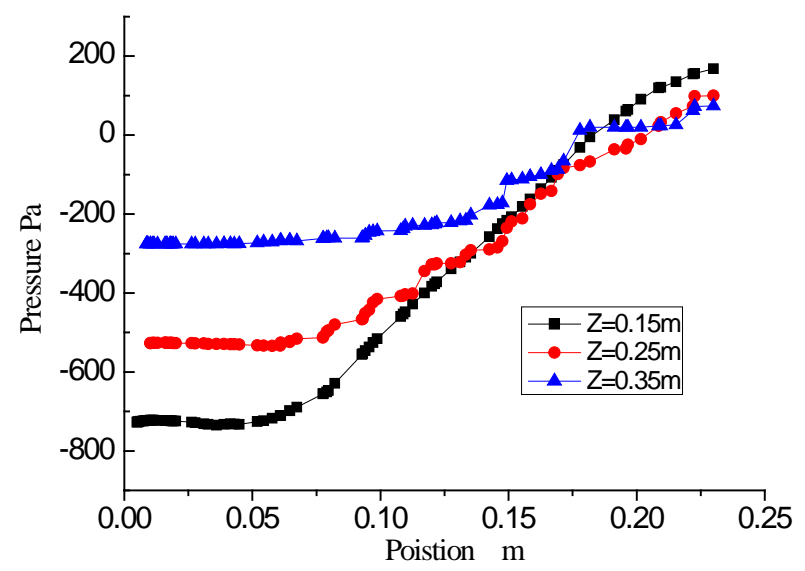

Fig 2 Pressure profile

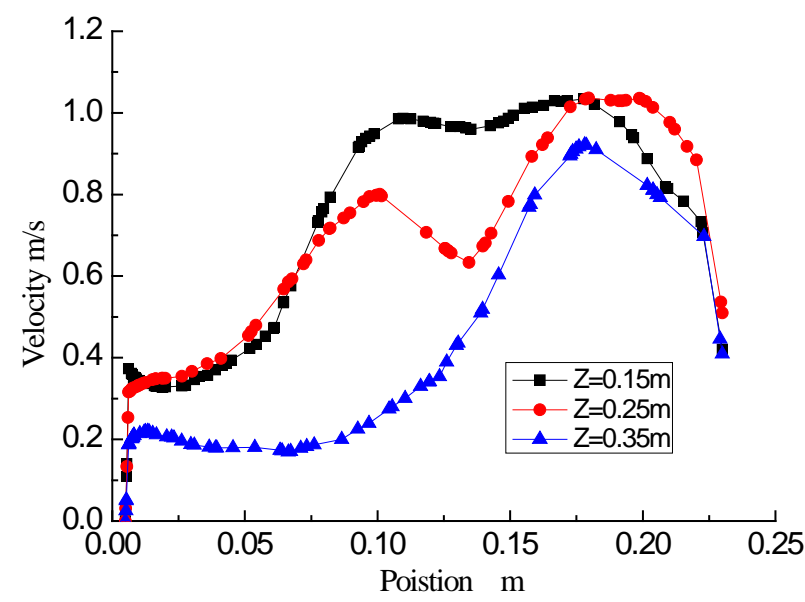

Fig 3 Velocity profile

Velocity distributions and vector distributions after mixing 1s and 10s are shown in Fig.4 and Fig.5. With the mixing time increasing, mixing domain gradually increases from the bottom to the top of the stirred tank. Annular mixing domain occurs almost from top to bottom of the end of the blade. After continuous mixing, solution is performed. Vector distributions at different time are in Fig.6. It can be seen that vortexes near the blade increase with the mixing. The surrounding fluid rotates with the rotations of the small vortexes, then vortex distribution regional presented. With the enhanced motion of the vortex, fluid far away from the blade domain mixes with the surrounding fluid. It verifies that the blade whirls to drive the fluid around to form a large number of vortexes. The motion and development of these vortexes enhance the mixing between the materials and the liquid.
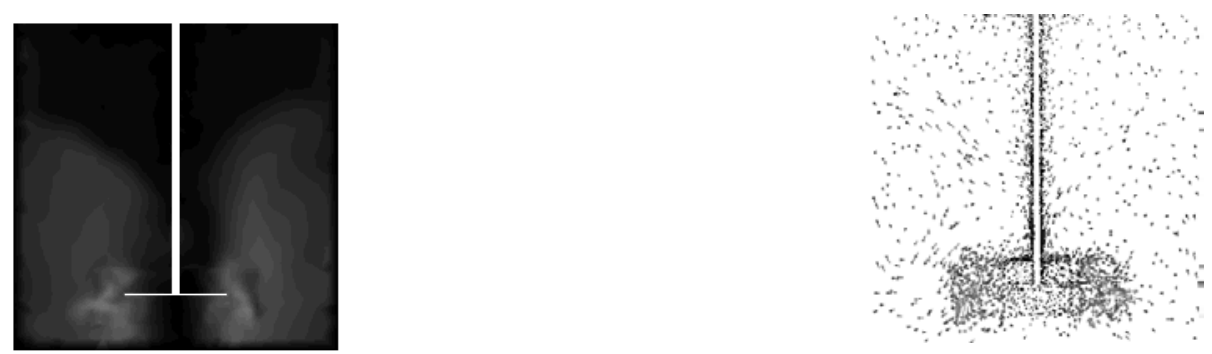

Fig 4 The velocity profile and vector profile at $1 \mathrm{~s}$ 

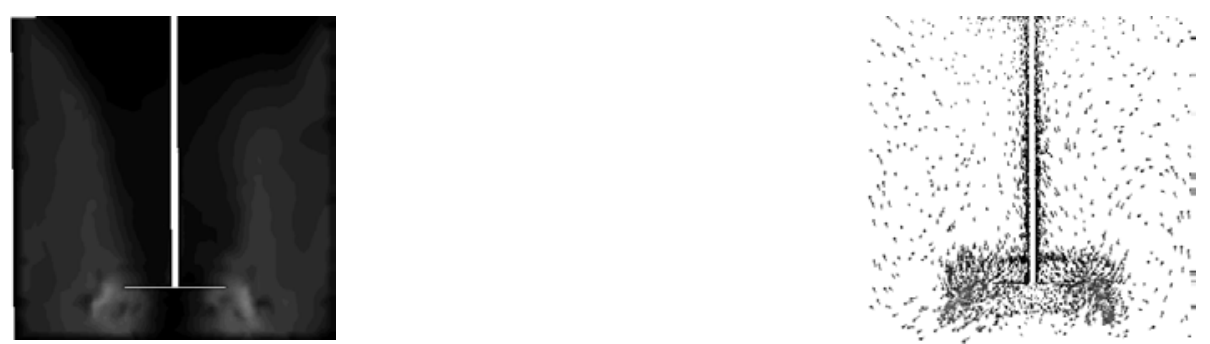

Fig 5 The velocity profile and vector profile at 10s
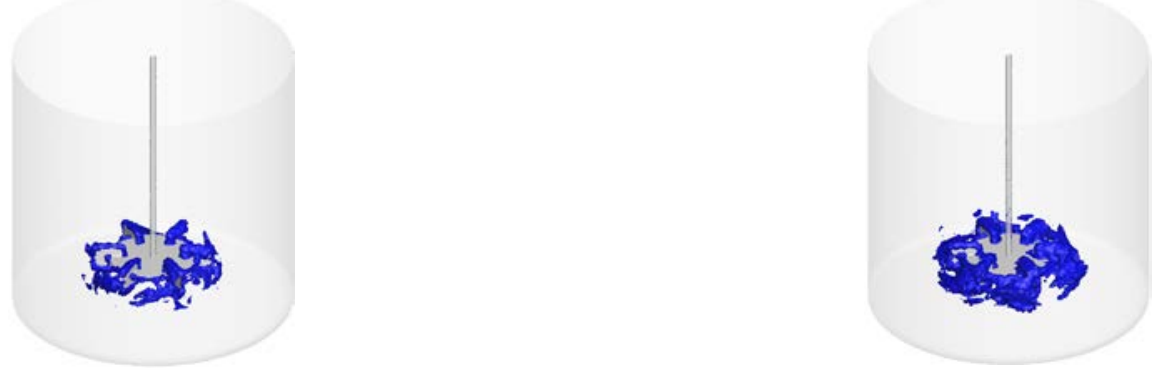

Fig 6 Vector profiles at different time

\section{Conclusions}

Flows of the stirred tank at different time are analyzed by the large eddy simulation method. The results show that the flow of six-blade ruston turbine is well and velocity distribution is uniform which is good for back-mixing and is unlikely to generate the cavitation. The flow velocity near the blade is obviously higher than that far away from the blade which shows that with the longer distance from the blade, mixing effect is weaker. The low pressure flow characteristic appears in the area near the blade. After squeezed to this area, the high pressure flow is mixed in the blade. The blade whirls to drive the fluid around to form a large number of vortexes. The motion and development of these vortexes enhance the mixing between the materials and the liquid.

\section{Acknowledgements}

This work was financially supported by the research foundation of Qingdao Science and Technology Development (13-1-4-245-jch)

\section{References}

[1] Lunden M, Stenberg O, Andersson B., Evaluation of a method $\mathrm{f}$ or measuring mixing time using numerical simulation and experimental data. Chem Eng Commun, Vol. 139(1995) , p. 115-136.

[2] Schmalzriedt S, Reuss M., Application of computational fluid dynamics to simulations of mixing and biotechnical conversion processes in stirred tank bioreactors. Proceedings of $9^{\text {th }}$ Europe Conference on Mixing. Nancy: GFGP, 1997.171-178.

[3] Jaworski Z, Bujalski W, Otiomo N., CFD study of homogenization with dual Rushton turbines: Comparison with experimental results. Tr ans IChemE, Vol. 78A(2000), p. 327-333. 